\title{
TTR
}

Traduction, terminologie, re?daction

\section{Hans J. Vermeer (1996). Übersetzen als Utopie. Die Übersetzungstheorie des Walter Bendix Schoenflies Benjamin. Heidelberg, TEXTconTEXT (Band 3)}

\section{Nadja Grbić et Michaela Wolf}

Volume 12, numéro 2, 2e semestre 1999

Poésie, cognition, traduction II - Autour d'un poème de W. H. Auden

Poetry, Cognition, Translation II — On a Poem by W. H. Auden

URI : https://id.erudit.org/iderudit/037383ar

DOI : https://doi.org/10.7202/037383ar

Aller au sommaire du numéro

Éditeur(s)

Association canadienne de traductologie

ISSN

0835-8443 (imprimé)

1708-2188 (numérique)

Découvrir la revue

Citer ce compte rendu

Grbić, N. \& Wolf, M. (1999). Compte rendu de [Hans J. Vermeer (1996).

Übersetzen als Utopie. Die Übersetzungstheorie des Walter Bendix Schoenflies

Benjamin. Heidelberg, TEXTconTEXT (Band 3)]. TTR, 12(2), 199-200.

https://doi.org/10.7202/037383ar d'utilisation que vous pouvez consulter en ligne.

https://apropos.erudit.org/fr/usagers/politique-dutilisation/ 
Hans J. Vermeer (1996). Übersetzen als Utopie. Die Übersetzungstheorie des Walter Bendix Schoenflies Benjamin. Heidelberg, TEXTconTEXT (Band 3)

Vermeer décrit la théorie de la traduction de Benjamin comme une utopie constituée par des hypothèses mythiques et des réminiscences de la " Geistesgeschichte ", qu'il place dans l'histoire des idées à la suite du siècle des Lumières (l'éducation de l'homme) et du Romantisme précoce (amplification des langues), lequel est, selon lui, étroitement lié à la tradition judaïque. Vermeer tente, dans cet ouvrage, de situer Benjamin dans le contexte de la théorie de la traduction. Le livre est divisé en trois parties. Dans la première partie, Vermeer se consacre à l'interprétation de la philosophie du langage de Walter Benjamin. Dans la deuxième, il traite des réflexions benjaminiennes autour de la reproductibilité de l'art, de son interprétation de la conceptualisatin des idées chez Platon ainsi que de son intérêt pour la monadologie. Dans la trosième partie, il étudie de façon détaillée sa théorie de la traduction en analysant son cadre théorique ainsi que les activités de W. Benjamin en 
tant que traducteur. Ce n'est qu'à la fin que Vermeer ajoute ses réflexions sur l'ouvrage d'Alfred Hirsch "Studien zum Sprach und Übersetzungsdenken Walter Benjamins und Jacques Derridas " (Munich, 1995), dont il ne disposa qu'après l'achèvement de son livre.

Selon Benjamin, la langue pure, paradisiaque, originelle est capable de saisir la substance des choses. Pourtant, dans la tradition judaïque, le déchirement des langues après la chute n'est pas connoté comme une dégradation. Cette tradition impose plutôt le devoir à l'homme d'achever la Création, afin que la langue soit reconstituée dans son unité et qu'elle redevienne parfaite. Ceci peut être réalisé aussi par la traduction. Car plus on traduit, plus les langues seront amplifiées et homogénéisées - jusqu'à la langue pure, dans laquelle tout peut être exprimé. La créativité et l'imagination mènent à la destitution de l'original et, par conséquent, à l'« inexprimabilité » qui prépare la perfection. Ce caractère « inexprimable » offre la possibilité de multiplier les interprétations d'un texte, de multiplier les contenus et d'atteindre cette " langue pure ", susceptible de tout exprimer. La solution consiste à traduire de façon à ce qu'aucun texte ne puisse se faire, mais que la fragmentarisation, la destruction de la forme, la littéralité en surgissent. Dans le non-sens tout reste à interpréter, l'original survivant aussi dans la traduction. Toujours selon Vermeer, la théorie de la traduction de Benjamin est cohérente dans le cadre de son « utopie judaïque et évolutionnaire de l'histoire » (p. 197), bien qu'elle ne vaille que sous les conditions idéalisantes et fortement réductrices d'un monde non réel. Car la rédemption de W. Benjamin par l'“ inexprimable " implique une dés-individualisation qui se trouve à l'opposé de la pluralité des réceptions ou des interprétations possibles. En ce sens, la théorie benjaminienne reste une utopie. 